\title{
Sevoflurano e desflurano sobre o ritmo cardíaco de cães tratados com infusão contínua de doses crescentes de adrenalina
}

\author{
Sevoflurane and desflurane in cardiac rhythm of dogs treated with increasing doses of epinephrine
}

\author{
Newton Nunes ${ }^{1}$ Márlis Langenegger de Rezende ${ }^{2}$ Paulo Sérgio Patto dos Santos $^{2}$ Lilia Wang ${ }^{3}$
}

\section{RESUMO}

Os anestésicos inalatórios sensibilizam o miocárdio ou seu sistema de condução à ação das catecolaminas endógenas elou exógenas, predispondo o animal às arritmias cardíacas. Dentre os anestésicos voláteis, o sevoflurano e o desflurano são fármacos relativamente recentes e, embora sejam dotados de características relacionadas a não sensibilização do miocárdio às catecolaminas, desconhecem-se estudos que comparem suas eventuais propriedades antiarritmogênicas. Com o objetivo de estudar, comparativamente, o comportamento do ritmo cardíaco e observar eventuais bloqueios atrioventriculares em cães anestesiados pelo sevoflurano e desflurano $e$ submetidos à infusão contínua de adrenalina, foram utilizados 20 animais adultos, os quais foram separados em dois grupos de igual número (G1 e G2). Aos cães do G1, foi administrado propofol, na dose média de $10 \mathrm{mg}^{.1 g^{-1}}$; em seguida os animais receberam sevoflurano, a 1,5CAM. Decorridos 30 minutos do início da administração do anestésico volátil, iniciou-se a infusão de adrenalina na dose de $1 \mu \mathrm{g} . \mathrm{kg}^{-1} \cdot \mathrm{min}^{-1}$. A cada 10 minutos, a dose da catecolamina foi acrescida em uma unidade, cessando-se a administração em $6 \mu \mathrm{g} \cdot \mathrm{kg}^{-1} \cdot \mathrm{min}^{-1}$. Para o G2, empregou-se a mesma metodologia, substituindo-se o sevoflurano pelo desflurano, administrado a 1,5CAM. A cada dose de adrenalina, foi feita contagem de batimentos ventriculares ectópicos, bem como a observação de bloqueios atrioventriculares. Os achados foram tratados pelos métodos estatísticos de Análise de Perfil e Kruskall-Wallis. Os resultados permitiram concluir que o desflurano minimiza de maneira mais eficiente a arritmia induzida pela adrenalina, além de reduzir a incidência de bloqueios atrioventriculares.

Palavras-chave: arritmia, adrenalina, desflurano, sevoflurano.

\section{ABSTRACT}

The volatile anesthetics increase the sensibility of the myocardium or its conduction system to the action of endogenous and/or exogenous epinephrine, predisposing the animal to cardiac arrhythmias. Among the volatile anesthetics, sevoflurane and desflurane are quite recent drugs and even though they have antiarrhythmogenic characteristics, there aren't studies comparing these properties. The aim of this work was to study comparatively the cardiac rhythm and observe occasional atrioventricular blockages in dogs anesthetized with desflurane and sevoflurane, receiving increasing doses of epinephrine. 20 healthy adult male and female mongrel dogs were used. The animals were separated in two groups (G1 and G2). In G1, anesthesia was induced with propofol $\left(10 \mathrm{mg}^{-1} \cdot \mathrm{kg}^{-1} \mathrm{IV}\right)$ and maintained with sevoflurane (1.5 MAC). Thirty minutes after the begin of volatile anesthetic administration, the epinephrine infusion was initiated at a dose of $1 \mu \mathrm{g} . \mathrm{kg}^{-1} \cdot \mathrm{min}^{-1}$. At each 10 minutes the dose was increased in $1 \mu \mathrm{g} . \mathrm{kg}^{-1} . \mathrm{min}^{-1}$ until $6 \mu \mathrm{g} . \mathrm{kg}^{-1} . \mathrm{min}^{-1}$. For G2, the same methodology was used, except that sevoflurane was replaced by desflurane (1.5 MAC). At each epinephrine dose, the non-sinusal cardiac beats were counted and the occurrence of atrioventricular blockages was evaluated. The numerical data were submitted to Profile analysis and Kruskall-Wallis. The results allow to conclude that desflurane reduces in a more efficient way the arrhythmia produced by epinephrine and also the occurrence of atrioventricular blockages.

Key words: arrhythmia, epinephrine, desflurane, sevoflurane.

\section{INTRODUÇÃO}

O uso da anestesia inalatória difundiu-se devido principalmente à segurança e à facilidade de controle da profundidade anestésica. Entretanto, os anestésicos inalatórios podem promover um aumento da automaticidade do miocárdio e, conseqüentemente, a propagação de impulsos provenientes de focos ectópicos, especialmente no ventrículo. Eles sensibilizam o miocárdio à ação arritmogênica das

${ }^{1}$ Professor Assistente, Doutor, Depto. de Clínica e Cirurgia Veterinária. Faculdade de Ciências Agrárias e Veterinárias, UNESP, 14870-000, Jaboticabal, SP. E-mail: newton@ fcav.unesp.br. Autor para correspondência.

${ }^{2}$ Pós-graduandos do Curso de Doutorado em Cirurgia Veterinária, Área de Concentração em Cirurgia, Faculdade de Ciências Agrárias e Veterinárias, UNESP, Campus de Jaboticabal, SP.

${ }^{3}$ Médico Veterinário Autônomo, São Paulo - SP. 
catecolaminas endógenas e/ou exógenas, sendo este efeito mais marcante na presença do halotano (KATZ \&BIGGER, 1970).

As arritmias cardíacas incluem anormalidades na freqüência cardíaca, no ritmo, no local de origem do impulso cardíaco, na despolarização atrial ou ventricular, e podem ser atribuídas a desordens na geração e/ou condução do impulso elétrico (HOFFMAN \& CRANEFIELD, 1964). A depressão na condução de resposta rápida e reentrada de impulsos excitatórios, são fenômenos elétricos anormais que resultam em estado patológico. Estas alterações são comumente originadas devido à hipóxia, isquemia, desequilíbrio eletrolítico e ainda à administração de certos fármacos (ATLEE \& BOSNJAK, 1990).

Dentre os anestésicos voláteis, o sevoflurano e o desflurano são fármacos relativamente recentes, que vêm sendo introduzidos na prática clínico-cirúrgica. Embora tais agentes sejam dotados de características relacionadas a não sensibilização do miocárdio às catecolaminas, são desconhecidos estudos que comparem suas eventuais propriedades antiarritmogênicas.

O sevoflurano é um anestésico volátil halogenado fluorinado, com peso molecular de 200; ponto de ebulição aos $59^{\circ} \mathrm{C}$, em nível do mar, e pressão de vapor equivalente a $160 \mathrm{mmHg}$ a $20^{\circ} \mathrm{C}$. Estudos recentes vêm mostrando que seus efeitos sobre o sistema cardiovascular parecem semelhantes aos do isoflurano, com poucas exceções. Segundo EBERT (1996), HETTRICK et al. (1996) e ROLF \& VANAKEN (1996), o sevoflurano parece não estar associado ao aumento da freqüência cardíaca, enquanto o isoflurano e o desflurano causam elevação significativa da mesma, quando em concentrações elevadas. Por outro lado, MUTOH et al. (1997), LOWE et al. (1996) e BERNARD et al. (1990) observaram aumento da freqüência cardíaca, com o uso do agente.

A elevação das concentrações de sevoflurano promove, ainda, diminuição progressiva da pressão arterial, de maneira semelhante ao que ocorre com os demais anestésicos voláteis (EBERT, 1996). Da mesma forma, foi observada por MUTOH et al. (1997), diminuição gradual na resistência vascular sistêmica, com o aumento dos níveis do anestésico.

Segundo HIKASA et al. (1996), a sensibilização do miocárdio aos efeitos arritmogênicos da adrenalina em felinos é significativamente menor com o sevoflurano do que com o halotano, não diferindo da observada com o uso de isoflurano. A dose de adrenalina requerida para indução de arritmias com o sevoflurano foi aproximadamente onze vezes maior do que a utilizada com o halotano.
O desflurano é um anestésico volátil halogenado fluorinado, com peso molecular de 168; ponto de ebulição aos $23,5^{\circ} \mathrm{C}$, em nível do mar e pressão de vapor equivalente a $664 \mathrm{mmHg}$ a $20^{\circ} \mathrm{C}$ (SMILEY, 1992), introduzido na prática clínico-cirúrgica em 1992 (WEISKOPF, 1995).

A administração do desflurano é associada ao aumento da atividade simpática, a qual atinge o pico máximo decorridos 5 minutos de exposição ao fármaco (PACENTINE et al., 1995). A ação simpática deve-se à existência de sítios receptores nas vias aéreas superiores, que respondem rapidamente à elevação da concentração. Esta resposta é resultante da ação irritante direta sobre a mucosa (MUZI et al., 1996). Porém, CIOFOLO \& REIZ (1999) constataram que o aumento da atividade simpática no homem parece estar relacionado à ativação central e não à irritação das vias aéreas superiores. No sistema cardiovascular, o desflurano possui a capacidade de diminuir a pressão arterial e a resistência vascular sistêmica, embora promova aumento da freqüência cardíaca (CLARKE et al., 1996), ocorrendo, ainda, retardo dose dependente na condução elétrica atrial e ventricular (SANTOS et al., 2000).

No homem, não foram encontradas evidências de que o desflurano induza à isquemia do miocárdio ou de que o fármaco aumente a taxa de mortalidade em pacientes com doenças coronárias (WARLTIER \& PAGEL, 1992). O agente volátil produz, também, propriedades inibitórias sobre arritmias ventriculares espontâneas, após infarto do miocárdio (NOVALIJA et al., 1998).

A contratilidade do miocárdio é deprimida pelo desflurano e o mesmo não difere do isoflurano quanto à sensibilização do músculo cardíaco, em pacientes submetidos a doses exógenas de adrenalina (NAVARRO et al., 1994).

Na tentativa de minimizar o risco anestésico, o conhecimento das características e propriedades dos fármacos é fundamental. Desta forma, objetivou-se avaliar, comparativamente, os efeitos sobre o ritmo cardíaco e eventuais bloqueios atrioventriculares em cães anestesiados pelo sevoflurano e desflurano, submetidos à infusão contínua de doses crescentes de adrenalina.

\section{MATERIAL E MÉTODOS}

Foram utilizados 20 cães (Canis familiaris, LINNAEUS, 1758), entre machos e fêmeas, sem raça definida, adultos, considerados sadios, evitando-se fêmeas prenhes ou em estro, fornecidos pelo canil do Hospital Veterinário "Governador Laudo Natel”, da 
Faculdade de Ciências Agrárias e Veterinárias - FCAV/ UNESP, Campus de Jaboticabal, SP.

Os animais foram distribuídos, aleatoriamente, em dois grupos de 10 (G1 e G2) e, previamente ao início do protocolo experimental, submetidos a avaliação eletrocardiográfica, de tal modo que os que apresentaram quaisquer alterações foram imediatamente descartados.

Aos animais do G1, foi administrado propofol, por via intravenosa, na dose média de 10 $\mathrm{mg} / \mathrm{kg}$ até a perda do reflexo laringotraqueal. Ato contínuo, os cães foram intubados com sonda de Magill e submetidos à anestesia inalatória sendo a sonda acoplada ao equipamento para administração de anestésico volátil em circuito valvular tipo "semifechado"a.

Cada cão foi mantido, sob anestesia, com sevoflurano $^{\text {b }}$, diluído em oxigênio com fluxo de $30 \mathrm{ml} /$ $\mathrm{kg} / \mathrm{min}$, na concentração alveolar mínima (CAM) de 1,5 , mensurada no ar expirado, por meio de analisador de gases ${ }^{c}$. Trinta minutos decorridos do início da administração do agente volátil, foi iniciada a aplicação, por meio de bomba de infusão ${ }^{\mathrm{d}}$, por via intravenosa, de adrenalina ${ }^{\mathrm{e}}$ a $0,02 \mathrm{mg} \cdot \mathrm{ml}^{-1}$, diluída em solução salina a $0,9 \%$ na dose inicial de $1 \mu \mathrm{g} \cdot \mathrm{kg}^{-1}$.minuto ${ }^{-1}$. A cada 10 minutos do início da administração da catecolamina, esta dose foi acrescida em uma unidade, cessando o incremento em $6 \mu \mathrm{g} \cdot \mathrm{kg}^{-1} \cdot$ minuto $^{-1}$, sendo a última dose administrada durante 10 minutos.

Para os animais do G2, empregaram-se os mesmos procedimentos, substituindo-se o sevoflurano pelo desflurano ${ }^{\mathrm{f}}$, administrado a 1,5 CAM, com o uso de vaporizador ${ }^{\mathrm{g}}$ microprocessado e calibrado para o agente. Os animais permaneceram sob monitoramento cardíaco contínuo desde a indução anestésica até o final do período experimental.

A cada dose de adrenalina, foi mensurada a quantidade de batimentos cardíacos de origem não sinusal. A variável foi obtida por observação da onda eletrocardiográfica em eletrocardiógrafo ${ }^{\mathrm{h}}$ computadorizado, na derivação D2, a partir da primeira dose de adrenalina, até o final da administração da catecolamina. Os momentos (M1 a M6) referem o número total de batimentos cardíacos de origem não sinusal, correspondentes às doses crescentes de adrenalina $\left(1,2,3,4,5,6 \mu \mathrm{g} \cdot \mathrm{kg}^{-1} \cdot\right.$ minuto $^{-1}$, respectivamente). Cada momento teve a duração de 10 minutos. Complementarmente, em ambos os grupos, foram também observadas a quantidade de bloqueios átrio-ventriculares de primeiro, segundo e terceiro graus.

A avaliação estatística foi efetuada por meio da Análise de Perfil (MORRISON, 1967; CURI,
1980), com $\mathrm{p}<5 \%$. Devido aos elevados coeficientes de variação encontrados, os achados numéricos foram, complementarmente, tratados pelo método não paramétrico de Kruskall-Wallis, considerando-se o valor de $\mathrm{p}<10 \%$.

\section{RESULTADOS}

A análise de perfil mostrou que as curvas obtidas dos grupos apresentaram perfis iguais e no mesmo nível estatístico. Não foram observadas diferenças entre os momentos em cada grupo. A contagem de figuras eletrocardiográficas anormais mostrou que a média em M3 foi significativamente maior no G1. Entretanto, com o método não paramétrico de Kruskall-Wallis, pôde-se identificar diferenças nos valores médios entre os grupos também em M4, ocasião em que a média do G1 foi superior à do G2. (Tabela 1).

Os resultados referentes à porcentagem de ocorrência de bloqueios atrioventriculares de $1^{\circ}, 2^{\circ} \mathrm{e}$ $3^{\circ}$ graus ao longo dos momentos foi maior nos animais submetidos à anestesia pelo sevoflurano (G1) do que nos submetidos à anestesia pelo desflurano (G2), exceto em M1, em que se observou a mesma porcentagem (30\%) de bloqueios atrioventriculares de $2^{\circ}$ grau em ambos os grupos. Nos demais momentos, os bloqueios atrioventriculares de $2^{\circ}$ grau, apresentaram seus valores de forma decrescente até M3 (10\%) e M2 (10\%), para G1 e G2, respectivamente. Com relação aos bloqueios atrioventriculares de $3^{\circ}$ grau, os mesmos não foram observados nos animais anestesiados pelo desflurano, enquanto que no grupo submetido ao sevoflurano, constatou-se a mesma porcentagem (10\%) em M1 e M2.

\section{DISCUSSÃO}

Relativamente ao número de batimentos ectópicos observados durante a anestesia pelo

Tabela 1 - Variação dos valores médios e desvios padrão de batimentos ventriculares ectópicos, em cães anestesiados com sevoflurano (G1) ou desflurano (G2) e submetidos a doses crescentes de adrenalina.

\begin{tabular}{lllllll}
\hline & M1 & M2 & M3 & M4 & M5 & M6 \\
\hline G1 & $9 \pm 20$ & $37 \pm 67$ & $58 \pm 82^{\mathrm{A} *}$ & $28 \pm 48^{*}$ & $9 \pm 18$ & $24 \pm 76$ \\
G2 & $8 \pm 25$ & $6 \pm 19$ & $0 \pm 0^{\mathrm{B} *}$ & $2 \pm 6^{*}$ & $29 \pm 86$ & $37 \pm 107$ \\
\hline
\end{tabular}

Médias seguidas de letras maiúsculas diferentes, nas colunas, indicam diferença estatística significativa pela Análise de Perfil $(\mathrm{p}<0,05)$.

Médias seguidas de asteriscos, nas colunas, indicam diferença estatística significativa pelo método de Kruskall-Wallis $(\mathrm{p}<0,1)$. 
sevoflurano, as médias obtidas, embora não estatisticamente significativas, apresentam tendência para uma arritmia de caráter dose-dependente, uma vez que os valores apresentados são crescentes até M3, correspondendo à infusão contínua de $3 \mu \mathrm{g} \cdot \mathrm{kg}^{-1} \cdot$ minuto $^{-1}$. A partir deste momento, mesmo com o aumento constante das doses da catecolamina, observa-se uma queda nas médias, que se prolonga até M5, sugerindo desta forma, saturação de receptores, provavelmente adrenérgicos (NUNES, 1995; REZENDE et al., 2000). Estes achados corroboram os encontrados por NAVARRO et al. (1994).

Nos animais tratados com desflurano, também não houve diferença estatística entre os momentos, mas foi observada elevação do número de batimentos não sinusais a partir de M5. Com doses maiores da catecolamina $\left(5 \mathrm{e} 6 \mu \mathrm{g} \cdot \mathrm{kg}^{-1} \cdot \mathrm{min}^{-1}\right)$, o potencial antiarritmogênico do desflurano mostrou-se menos eficiente, apresentando elevação da ocorrência de batimentos não sinusais. Dessa forma, o desflurano não parece dotado das mesmas propriedades que o sevoflurano, no que concerne à redução da quantidade de complexos ventriculares ectópicos, a partir de certa dose de adrenalina, como descrito por REZENDE et al. (2000). Por outro lado, pode-se observar que em concentrações menores de catecolamina (3 e $4 \mu \mathrm{g} \cdot \mathrm{kg}^{-1} \cdot \mathrm{min}^{-1}$ ), o desflurano apresentou potencial antiarritmogênico estatisticamente superior ao sevoflurano.

KITAA \& MITEMA (1994) descreveram, em cães, fibrilações ventriculares precedidas de taquicardia, nas quais três animais foram a óbito dentro de um minuto após a administração de adrenalina, constatando que o halotano sensibiliza o miocárdio à despolarização atrioventricular prematura induzida pela adrenalina (HIKASA et al., 1996). Tais achados corroboram a assertiva de que o sevoflurano e o desflurano são mais seguros que o halotano quanto à sensibilização do miocárdio às catecolaminas, uma vez que em nenhum momento, o número de batimentos não sinusais atingiu valores considerados de risco para os animais. Segundo TRANQUILLI et al. (1986), deve-se agir apenas quando o número de batimentos ectópicos superar 16 ocorrências, ou mais, por minuto.

De acordo com NOVALIJA et al. (1998), existe redução da atividade ventricular ectópica durante a administração do desflurano e sevoflurano, embora naquela ocasião os autores tenham encontrado valores mais favoráveis ao segundo anestésico, em detrimento do primeiro. A diferença marcante na metodologia empregada entre o presente experimento e aquele supracitado poderia explicar a pouca semelhança entre os resultados.
Os bloqueios atrioventriculares de $1^{\circ}, 2^{\circ} \mathrm{e}$ $3^{\circ}$ graus, foram observados com maior freqüência no G1, nos momentos M1, M2 e M3, não sendo observados nos demais. $\mathrm{O}$ aparecimento de bloqueio atrioventricular completo também foi descrito por MARUYAMA et al. (1998), com a utilização do sevoflurano e óxido nitroso durante indução lenta em paciente apresentando hipertensão, disfunção renal, e condução cardíaca prejudicada.

Embora HAMMOND et al. (1994) tenham relatado a ocorrência de bloqueios atrioventriculares com despolarizações ventriculares multifocais ectópicas como um dos efeitos colaterais do desflurano, cabe ressaltar que, por ocasião deste experimento, o desflurano foi capaz de impedir o aparecimento de bloqueios atrioventriculares de $3^{\circ}$ grau, ao contrário do observado com o uso de sevoflurano em M1 e M2. Mesmo o aparecimento de bloqueios de graus 1 e 2 ocorreu em menor porcentagem com o desflurano, quando comparado aos achados resultantes dos cães anestesiados com sevoflurano. Apesar da literatura ser omissa quanto a estas alterações eletrocardiográficas, em animais tratados com fármacos arritmogênicos e anestesiados com agentes voláteis de uso recente, é possível inferir que o desflurano, em maior grau que o sevoflurano, impede o retardo na condução atrioventricular ou seu bloqueio.

SANTOS et al. (2000) observaram retardo na condução atrioventricular, imputado ao aumento da impedância do miocárdio, determinada pelo desflurano. Tal achado pôde ser corroborado pela observação de bloqueios, principalmente de segundo grau, com menores doses de catecolamina. Entretanto a adrenalina, provavelmente devido ao seu potencial facilitador de despolarização do músculo cardíaco, foi capaz de atenuar, nos cães anestesiados pelo desflurano, o aumento da resistência ao impulso elétrico gerado no miocárdio superior. $\mathrm{O}$ mesmo pode ter ocorrido com os animais do G1, embora, com menores doses de catecolamina, o sevoflurano tenha favorecido o retardo na condução atrioventricular.

\section{CONCLUSÕES}

Frente aos resultados obtidos, foi possível concluir que ambos os anestésicos voláteis são seguros quanto à baixa incidência de figuras eletrocardiográficas anormais, em cães tratados com infusão contínua de adrenalina. Entretanto, o desflurano proporciona menor ocorrência destes achados, com doses menores de catecolamina, além de reduzir a porcentagem de bloqueios atrioventriculares. 


\section{FONTES DE AQUISIÇÃO}

a- OHMEDA mod Excel 210 SE - FAPESP 97/10668-4

b- Sevorane - Cristália Produtos Químicos e Farmacêuticos Ltda.

c- Monitor de Gases mod. RGM 5250 OHMEDA

d- Lifemed mod. FARS 600 - FAPESP 95/2763-1

e- Cloridrato de adrenalina 1/1000 - Laboratório Santista Ltda.

f- Suprane - Laboratórios Zêneca S.A.

g. OHMEDA mod. TEC 6 - FAPESP 97/10668-4

h. TEB mod. ECG-PC software versão 1.10 - FAPESP 95/ $2763-1$

\section{REFERÊNCIAS BIBLIOGRÁFICAS}

ATLEE, J.L.; BOSNJAK, Z.J. Mechanisms of cardiac disrhytmias during anesthesia. Anesthesiology, Hagerstown, v.72, n.2, p.347-367, 1990.

BERNARD, J.M. et al. Effects of sevoflurane and isoflurane on cardiac and coronary dynamics in chronically instrumented dogs. Anesthesiology, Hagerstown, v.72, n.4, p.659-62, 1990 .

CIOFOLO, M.J.; REIZ, S. Circulatory effects of volatile anesthetic agents. Minerva Anestesiologica, Turin, v.65 n.5, p.232-238, 1999 .

CLARKE, K.W. et al. Cardiopulmonary effects of desflurane in the dog during spontaneous and artificial ventilation. Research in Veterinary Science, London, v.61, p.82-86, 1996.

CURI, P.R. Análise de medidas repetidas em experimentos biológicos. Revista Brasileira de Estatística, São Paulo, v.41, n. 161, p.137-150, 1980 .

EBERT, T.J. Cardiovascular and autonomic effects of sevoflurane. Acta Anaesthesiologica Belgica, Brussels, v.47, n.1, p.15-21, 1996.

HAMMOND, R.A. et al. Desflurane in the dog; minimal alveolar concentration alone and in combination with nitrous oxide. Journal of American Veterinary Medical Association, Schaumburg, v.21, p.21-23, 1994.

HETTRICK, D.A.; PAGEL, P.S.; WARLTIER, D.C. Desflurane, sevoflurane, and isoflurane impair canine left ventricular-arterial coupling and mechanical efficiency. Anesthesiology, Hagerstown, v.85, n.2, p.404-413, 1996.

HIKASA, Y. et al. Ventricular arrhythmogenic dose of adrenaline during sevoflurane, isoflurane and halothane anaesthesia either with or without ketamine or thiopentone in cats. Research in Veterinary Science, London, v.60, n2, p.134-137, 1996

HOFFMAN, B.F.; CRANEFIELD, P.F. The physiological basis of cardiac arrhytmias. American Journal of Medicine, Newton, v.37, p.670-684, 1964.

KATZ, R.L.; BIGGER, J.T. Cardiac arrhytmia during anesthesia and operation. Anestesiology, Hagerstown, v.33, p.193213,1970 .
KITAA, J.M.A.; MITEMA, E.S. Effects of antiarrhythmic drugs (Verapamil, propanolol and lignocaine) on the electrocardiogram and haematology in adrenaline-induced arrhythmias in dogs anaesthetized with halothane. British Veterinary Journal, London, v.150, n.4, p.365-376, 1994.

LOWE, D. et al. Influence of volatile anesthetics on left ventricular afterload in vivo. Differences between desflurane and sevoflurane. Anesthesiology, Hagerstown, v.85, n.1, p.112-120, 1996 .

MARUYAMA, K. et al. Slow induction with sevoflurane was associated with complete atrioventricular block in a child with hypertension, renal dysfunction, and impaired cardiac conduction. Paediatric Anaesthesia, Basel, v.8, n.1, p.73$78,1998$.

MORRISON, D.F. Multivariate statistical methods. New York: MaC Grows Hill., 1967. 388p.

MUTOH, T. et al. Cardiopulmonary effects of sevoflurane, compared with halotane, enflurane, and isoflurane, in dogs. American Journal of Veterinary Research, Schaumburg, v.58, n.8, p.885-890, 1997.

MUZI, M. et al. Site(s) mediating sympathetic activation with desflurane. Anestesiology, Hagerstown, v.85, n.4, p.737747, 1996.

NAVARRO, R. et al. Humans anesthetized with sevoflurane or isoflurane have similar arrhythmic response to epinephrine. Anesthesiology, Hagerstown, v.80, n.3, p.545-549, 1994.

NOVALIJA, E. et al. Effects of desflurane, sevoflurane and halothane on postinfarction spontaneous dysrhythmias in dogs. Acta Anaesthesiologica Scandinavica, Copenhagen, v.42, n.3, p.353-357, 1998.

NUNES, N. Efeitos cardiocirculatórios da associação de quetamina e levomepromazina em cães (Canis familiaris) pré-tratados ou não pelo propanolol. 1995. 141f. Tese: (Doutorado em Medicina Veterinária) - Faculdade de Medicina Veterinária e Zootecnia, Universidade Estadual Paulista, Botucatu.

PACENTINE, G.G.; MUZI, M.; EBERT, T.J. Effects of fentanyl on sympathetic activation associated with the administration of desflurane. Anestesiology, Hagerstown, v.82, p.823-831, 1995

REZENDE, M.L. et al. Emprego da levomepromazina no bloqueio da arritmia induzida pela adrenalina em cães anestesiados pelo sevoflurano. Ciência Rural, Santa Maria, v.30, n.3, p.421-424, 2000.

ROLF, N.; VAN AKEN, H. The cardiovascular effects of sevoflurane. Anaesthesist, Heidelberg, v.45, n.Suppl 1, p.1421, 1996.

SANTOS, P.S.P. et al. Estudo eletrocardiográfico em cães tratados com concentrações decrescentes de desflurano. Ars Veterinária, Jaboticabal, v.16, n.1, p.19-21, 2000

SMILEY, R.M. An overview of induction and emergence characterictics of desflurane in pediatric, adult, and geriatric patients. Anesthesia and Analgesia, New York, v.75, p.3846, 1992. 
TRANQUILLI, W.J. et al. Alteration in the arrythmogenic dose of epinephrine (ADE) following sylazine administration to halothane-anesthetized dogs. Journal of Veterinary Pharmacology and Therapeutics, Oxford, v.9, n.2, p.198203, 1986.

WARLTIER, D.C.; PAGEL, P.S. Cardiovascular and respiratory actions of desflurane: is desflurane different from isoflurane? Anesthesia and Analgesia, New York, v.75, p.17-31, 1992.

WEISKOPF, R.B. Cardiovascular effects of desflurane in experimental animals and volunteers. Anaesthesia, London, v.50, p.14 -17, 1995 . 